\title{
Avaliação cefalométrica do espaço aéreo posterior em pacientes com oclusão Classe II submetidos à cirurgia ortognática
}

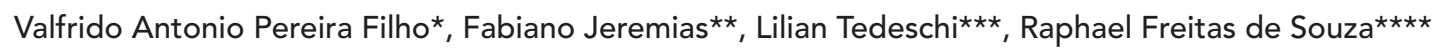

\begin{abstract}
Resumo
Objetivo: o avanço maxilomandibular é um método cirúrgico comumente usado no tratamento de pacientes acometidos pela Síndrome da Apnéia Obstrutiva do Sono (SAOS) e portadores de anormalidades anatômicas identificáveis neste complexo, que estreitam e/ou obstruem o espaço aéreo. $\mathrm{O}$ intuito deste estudo foi analisar variações cefalométricas do espaço aéreo faríngeo em indivíduos Classe II de Angle, após a cirurgia ortognática. Metodologia: a amostra consistiu de telerradiografias laterais equivalentes aos períodos pré e pós-operatório de 30 indivíduos, divididos no grupo com avanço cirúrgico mandibular (n=15) e no grupo com avanço maxilomandibular $(\mathrm{n}=15)$. Os parâmetros cefalométricos usados permitiram avaliar o espaço aéreo posterior em 3 níveis: a hipofaringe (PFI-V), a orofaringe (PFM-PM, PFM-PO, PFM-U, PFM-Up) e a nasofaringe (PFM-PN, pm-PFS). A análise esquelética foi na base do crânio (N-S-Ba) e na mandíbula (Ar-Go-Me). A média das diferenças entre os valores pré e pós-operatórios das mensurações lineares (mm) e angulares (graus) foi avaliada pelo teste $t$ pareado. Resultados e Conclusões: estatisticamente, não houve redução do espaço aéreo faríngeo pós-avanço cirúrgico. O que se observou foi que apenas PFM-PO e PFS-pM se mantiveram constantes e na maioria restante os valores aumentaram.
\end{abstract}

Palavras-chave: Cefalometria. Cavidade nasofaríngea. Oclusão Classe II. Cirurgia ortognática.

\section{INTRODUÇÃO}

O crescimento da base anterior do crânio e do complexo maxilomandibular pode produzir alterações no volume do espaço aéreo posterior e desordens respiratórias do sono, como o ronco, que poderão ocorrer se as paredes deste espaço estiverem em colapso ${ }^{5}$. A Síndrome da Apnéia
Obstrutiva do Sono (SAOS) representa o estágio mais severo, na qual os músculos são hipotônicos durante o sono e a estabilidade do espaço aéreo se torna dependente do tamanho da faringe e da relação desses tecidos ${ }^{19}$.

As manifestações clínicas da síndrome são: estreitamento ou obstrução do espaço aéreo superior,

* Professor Assistente-Doutor da Disciplina de Cirurgia e Traumatologia Buco-Maxilo-Facial da Faculdade de Odontologia de Araraquara - UNESP.

** Acadêmico e estagiário da Disciplina de Cirurgia e Traumatologia Buco-Maxilo-Facial da Faculdade de Odontologia de Araraquara - UNESP.

*** Cirurgiã-dentista, Especialista em Radiologia Odontológica e Imaginológica da Faculdade de Odontologia de Ribeirão Preto-USP.

**** Professor Assistente-Doutor do Departamento de Materiais Dentários e Prótese da Faculdade de Odontologia de Ribeirão Preto - USP. 
sono fragmentado e distúrbios cardiorespiratórios decorrentes de desordens respiratórias. As conseqüências cardiovasculares desta síndrome podem incluir hipertensão sistêmica, arritmias cardíacas, infarto do miocárdio e acidentes vásculo-cerebrais. Outros relatos incluem a falta de sono noturno completo, sonolência diurna, dor de cabeça matinal, ausência de sonhos, fadiga, boca seca, diminuição da libido e sintomas de depressão ${ }^{25}$.

$\mathrm{Na}$ etiologia da SAOS, fatores anatômicos e patofisiológicos estão implicados ${ }^{1}$ e com isso a avaliação radiográfica da face e do espaço aéreo tem recebido atenção considerável. Tanto técnicas radiográficas cefalométricas laterais quanto tomografias tridimensionais têm sido empregadas neste contexto. Mesmo a visão cefalométrica fornecendo uma figura dimensional limitada, tem tido o mérito de ser simples e mais facilmente avaliável do que a tomografia computadorizada ou a ressonância magnética ${ }^{2}$. A imagem do espaço aéreo superior é realizada para obter informações adicionais a respeito da anatomia do fluxo aéreo. Por exemplo, o exame clínico nem sempre revela uma deformidade facial, mas a cefalometria pode ajudar na determinação dos fatores anatômicos contribuintes para a síndrome. Esta informação pode ser usada para direcionar o tratamento, particularmente cirúrgico, e levar a resultados no tratamento ${ }^{7}$.

Estudos envolvendo cefalometria radiográfica em pacientes com a síndrome têm demonstrado que estes pacientes têm mandíbulas pequenas e retroposicionadas, espaço aéreo posterior estreito e palato mole e língua maiores do que o grupo controle, assim como osso hióide posicionado inferiormente e maxila retroposicionada ${ }^{24}$. Na SAOS, diferenças esqueléticas têm sido reportadas nos planos horizontais e verticais. Ântero-posteriormente, tanto a face quanto a base do crânio tendem a se retruir ${ }^{14,30}$ e o ângulo da base do crânio se reduz ${ }^{12}$. Este arranjo esquelético leva a uma redução no espaço respiratório. Retrusão mandibular adicional também pode ocorrer. Jamieson et al. ${ }^{12}$ descreveram um modelo Classe II, recentemente confirmado por Battagel e L'Estrange ${ }^{1}$ e Lowe et al. ${ }^{14}$ Até mesmo na ausência de desarmonia esquelética, onde a face é retroposicionada, o corpo da mandíbula pode ser pequeno ${ }^{1}$. No plano vertical, aumento na altura facial inferior e ângulos do plano maxilomandibular têm sido reportados ${ }^{14,30}$. Embora muitos trabalhos indiquem que exista uma morfologia esquelética típica nos indivíduos com $\mathrm{SAOS}^{4}$, estudos mais recentes sugerem que esta é uma visão simplista ${ }^{1,11,14}$.

Nos pacientes com anormalidades anatômicas identificáveis nos maxilares, resultante em estreitamento da cavidade nasofaríngea, a cirurgia ortognática se mostra como excelente opção de tratamento. $\mathrm{O}$ uso da cirurgia ortognática para tratar a síndrome se iniciou no final dos anos 70 , quando o avanço mandibular foi usado para reverter os sintomas da síndrome ${ }^{3}$. Desde então, este procedimento tem se tornado amplamente aceito ${ }^{22}$. O tratamento ortognático da síndrome pode envolver avanço da maxila, mandibula e mento. Esses três procedimentos podem ser realizados em combinação, contudo o avanço da maxila e mandíbula, com ou sem genioplastia, é o procedimento mais comum. O plano de tratamento é baseado na análise facial e cefalométrica craniofacial, que determina se a posição da maxila, mandíbula e mento é normal ou anormal. O procedimento requer rigor no planejamento précirúrgico e na técnica cirúrgica em si. As complicações pós-operatórias são mais comuns que as pré, porém não retardam o resultado final esperado ${ }^{6,11}$.

\section{PROPOSIÇÃo}

O objetivo do estudo é analisar as variações cefalométricas do espaço aéreo faríngeo em indivíduos com Classe II de Angle, após a cirurgia ortognática para correção de deformidade dentofacial.

\section{MATERIAL E MÉTODOS}

A amostra constou da análise de 30 casos de indivíduos Classe II de Angle submetidos à cirurgia ortognática, sendo 15 casos operados na mandíbula (MD) e 15 operados na maxila e mandíbu- 
la (MX/MD), obtidos do arquivo do Centro de Pesquisa e Tratamento das Deformidades BucoFaciais (CEDEFACE) de Araraquara.

A análise cefalométrica foi realizada por meio de telerradiografias laterais padronizadas, que representassem duas fases operatórias de cada caso, ou seja, uma pré-operatória e uma pós-operatória (mínimo de 6 meses). Tal procedimento foi realizado em duplicidade, por dois examinadores, em sala totalmente escura, exceto pela luz do negatoscópio, sobre o qual foram feitas as análises, após aderir papel transparente tipo Ultraphan às radiografias. Os pontos cefalométricos identificados e analisados constam no quadro $1^{10,27,28}$.

Os parâmetros cefalométricos usados (Fig. 1) permitiram avaliar o espaço aéreo posterior em 3 níveis: a hipofaringe (PFI-V), a orofaringe (PFM-

\begin{tabular}{cl}
\hline ENA & espinha nasal anterior \\
ENP & espinha nasal posterior \\
Pm & fissura pterigomaxilar \\
Ba & básio \\
U & ponta da úvula: ponto mais póstero-inferior da úvula \\
PFS & parede faríngea superior: intersecção entre a linha \\
& Pm-Ba e a parede posterior faríngea \\
PFM & parede faríngea média: intersecção entre a linha \\
& perpendicular em U e a parede posterior faríngea \\
PFI & parede faríngea inferior: intersecção entre a linha per- \\
& pendicular da valécula e a parede posterior faríngea \\
valécula & intersecção da epiglote e da base da língua \\
Me & mento \\
Go & gônio \\
IIS & incisal do incisivo superior \\
III & incisal do incisivo inferior \\
PMS & primeiro molar superior \\
PMI & primeiro molar rinferior \\
PM & plano mandibular \\
PO & plano oclusal \\
PN & plano nasal \\
N & násio \\
S & sela \\
\hline
\end{tabular}

Quadro 1 - Pontos cefalométricos utilizados.
PM, PFM-PO, PFM-U, PFM-Up) e a nasofaringe (PFM-PN, pm-PFS). A análise esquelética foi realizada na base do crânio (N-S-Ba) e na mandíbula (Ar-Go-Me).

A média das diferenças entre os valores pré e pós-operatórios das mensurações lineares ( $\mathrm{mm}$ ) e angulares (graus) foi avaliada pelo teste $t$ pareado.

\section{RESULTADOS E DISCUSSÃO}

Estatisticamente, não houve redução do espaço aéreo faríngeo, após avanço cirúrgico. $\mathrm{O}$ que se observou foi que apenas 2 medidas se mantiveram constantes e na maioria restante os valores aumentaram (Tab. 1).

Tabela 1 - Média das diferenças entre os valores pré e pós-operatórios das medidas lineares $(\mathrm{mm})$ e angulares (graus) e os resultados do teste t pareado, (a.m.= avanço mandibular; a.m.m. = avanço maxilomandibular).

\begin{tabular}{ccccc}
\hline & média & $\begin{array}{c}\text { desvio- } \\
\text { padrão }\end{array}$ & t & p \\
\hline V-PFI, a.m. & $-1,3$ & 2,3 & $-2,2$ & $0,049^{*}$ \\
V-PFI, a.m.m. & $-1,9$ & 2,3 & $-3,2$ & $0,007^{* *}$ \\
PFM-PM, a.m. & $-0,9$ & 2,5 & $-1,4$ & 0,171 n.s. \\
PFM-PM, a.m.m. & $-2,8$ & 3,2 & $-3,3$ & $0,005^{* *}$ \\
PFM-PO, a.m. & $-0,1$ & 3,5 & $-0,1$ & 0,898 n.s. \\
PFM-PO, a.m.m. & $-1,5$ & 5,4 & $-1,1$ & 0,308 n.s. \\
PFM-U, a.m. & $-0,4$ & 2,4 & $-0,7$ & 0,513 n.s. \\
PFM-U, a.m.m. & $-1,6$ & 2,2 & $-2,8$ & $0,014^{*}$ \\
PFM-Up, a.m. & $-1,4$ & 2,4 & $-2,2$ & $0,042^{*}$ \\
PFM-Up, a.m.m. & $-1,7$ & 1,9 & $-3,5$ & $0,003^{* *}$ \\
PFM-PN, a.m. & $-0,6$ & 2,4 & $-1,0$ & 0,343 n.s. \\
PFM-PN, a.m.m. & $-2,3$ & 2,0 & $-4,6$ & $0,000^{* *}$ \\
PM-PFS, a.m. & $-1,0$ & 2,8 & $-1,4$ & 0,183 n.s. \\
PM-PFS, a.m.m. & $-0,9$ & 3,0 & $-1,2$ & 0,269 n.s. \\
N-S-Ba, a.m. & $-0,8$ & 1,3 & $-2,4$ & $0,032^{*}$ \\
N-S-Ba, a.m.m. & $-0,5$ & 1,2 & $-1,6$ & 0,133 n.s. \\
Ar-Go-Me, a.m. & $-2,1$ & 3,1 & $-2,6$ & $0,020^{*}$ \\
\hline Pro-Me, a.m.m. & $-0,6$ & 6,7 & $-0,4$ & 0,726 n.s. \\
\hline PFerenca na sig & & &
\end{tabular}

n.s. = diferença não significante;

* significante $(p<0,05)$; ${ }^{* *}$ significante $(p<0,01)$. 


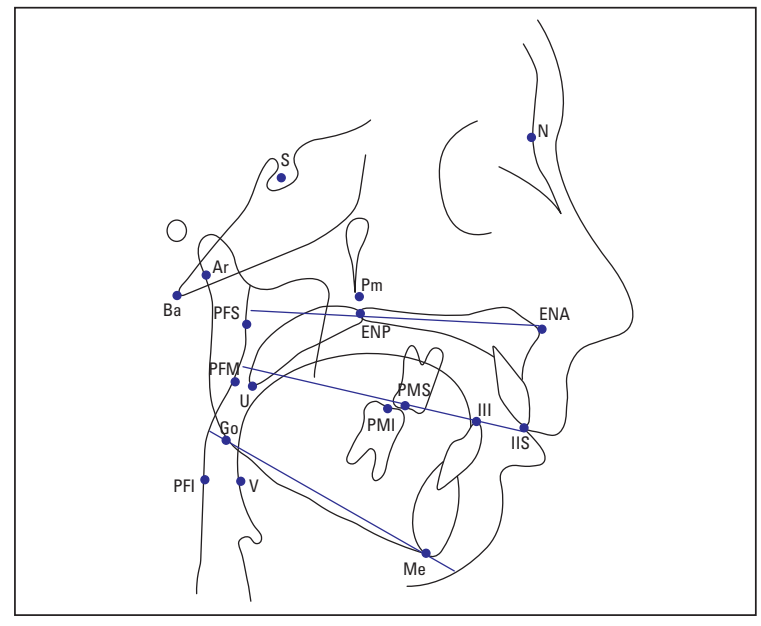

FIGURA 1 - Pontos cefalométricos e planos mandibular, oclusal e nasal usados no estudo.

A cefalometria radiográfica tem sido usada como ferramenta de mensuração no estudo do espaço aéreo faríngeo e da morfologia craniofacial, oferecendo vantagens consideráveis sobre outras técnicas, como baixo custo, exposição mínima à radiação, bem como permitindo análise simultânea da posição da cabeça, do osso hióide e da morfologia craniofacial ${ }^{17}$.

Os parâmetros cefalométricos usados neste estudo $^{10,27,28}$ permitiram avaliar o espaço aéreo posterior, pós-cirurgia ortognática, em três níveis: hipofaringe (PFI-V), orofaringe (PFM-PM, PFMPO, PFM-U, PFM-UP) e nasofaringe (PFM-PN, pm-PFS). Alguns estudos ${ }^{15,26}$ não correlacionaram nenhuma estrutura esquelética nas análises do espaço aéreo faríngeo, mas devido ao fato de que mudanças adaptativas ocorrem nos tecidos mole e duro após as osteotomias ${ }^{13,18}$, foi realizada análise esquelética na base do crânio (Násio-Sela-Básio) e na mandíbula (Articulare-Gônio-Mento).

Resultados pós-operatórios demonstraram aumento no espaço aéreo hipofaríngeo em $70 \%$ dos casos analisados, sendo que o ângulo PFI-V se elevou após a cirurgia ortognática, independente de o avanço ser restrito à mandíbula ou não, o que provavelmente reposicionou mais anteriormente a musculatura supra-hióidea e o osso hióide. Nos estudos de Riley et al. ${ }^{22}$ e De Ponte et al. ${ }^{5}$ também

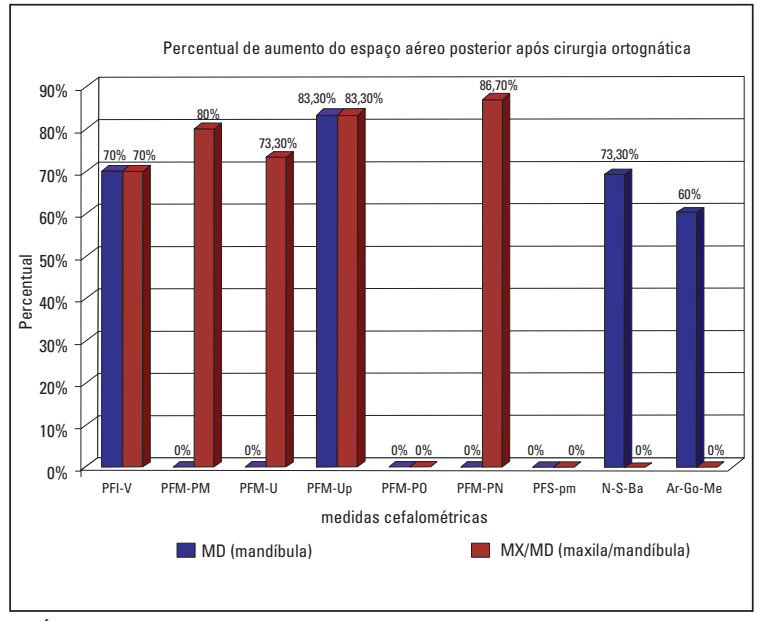

GRÁFICO 1 - Percentual de aumento do espaço aéreo posterior após cirurgia ortognática.

se constatou aumento significativo nesta região faríngea. Uma redução em PFI-V ocorreu em 7 pacientes e em apenas 2 os valores se mantiveram constantes.

Para Muto et al. ${ }^{17}$, anatomicamente, este espaço aéreo faríngeo (PFI-V) está circundado por tecido mole e pela mandíbula, dependendo tanto do volume de tecido, quanto do tamanho e posição mandibular. Mais importante é o fato de que a parede posterior deste osso pode mover-se ântero-posteriormente com alterações dos ângulos craniocervicais. Desta forma, um valor aumentado de PFI-V está associado a um grande reposicionamento anterior da mandíbula.

Quatro parâmetros cefalométricos foram utilizados para avaliar o espaço aéreo orofaríngeo. A medida linear PFM-UP teve o maior aumento (83,3\% dos casos), seguida de PFM-PM (80\% dos casos) e PFM-U (73,3\% dos casos). No caso das duas últimas, o aumento ocorreu apenas quando a intervenção cirúrgica foi bimaxilar (maxilomandibular), enquanto na primeira o aumento independia do avanço ser restrito à mandíbula ou não. A medida PFM-PO se manteve constante, estatisticamente. Os achados do presente estudo, contrastam com os de De Ponte et al. ${ }^{5}$, que verificaram aumento pós-operatório em 75\% dos pacientes para PFM-PM, 57\% para PFM-PO e PFM-U e 37\% 
para PFM-UP. De acordo com Schendel et al. ${ }^{23} \mathrm{e}$ Hochban et al. ${ }^{11}$, o aumento no PFM-U e PFM-UP se deve ao avanço do palato mole e faringe, após o movimento maxilar.

O tratamento das deformidades dentofaciais, com osteotomia maxilar tem um efeito na morfologia orofaríngea. Conseqüentemente, o avanço mandibular tem sido usado com sucesso para aumentar o espaço aéreo em portadores da Síndrome da Apnéia Obstrutiva do Sono ${ }^{15,22}$. Por outro lado, o recuo mandibular reduz o tamanho do espaço aéreo faríngeo ${ }^{29} \mathrm{e}$, em alguns casos raros, pode desenvolver distúrbio respiratório do sono ${ }^{21}$.

As alterações na dimensão do espaço aéreo posterior variam conforme a quantidade de avanço cirúrgico realizado. Nos achados de Mehra et al. ${ }^{15}$ ocorreu um ligeiro aumento nas dimensões aéreas retropalatal e retrolingual, possivelmente devido ao avanço mandibular ter sido pequeno. Para Reiche-Fischel e Wolford ${ }^{20}$ uma porcentagem de aumento maior nas dimensões aéreas orofaríngea foi observada aos $10 \mathrm{~mm}$ de avanço. Após tal valor, o espaço continua a aumentar em dimensão, mas com proporção menor relacionada à quantidade de avanço mandibular.

A posição da cabeça e do pescoço também influencia nas mudanças das dimensões aéreas, que ocorrem após a cirurgia de avanço maxilomandibular, como tem sido demonstrado em estudos clínicos e experimentais ${ }^{9,17}$. Quanto ao espaço nasofaríngeo, o valor de PFM-PN aumentou em $86,7 \%$ dos pacientes e diminuiu em $13,3 \%$, apenas quando a intervenção foi bimaxilar. Nos achados de De Ponte et al. ${ }^{5}$, o aumento foi constatado em $39 \%$ dos pacientes, o decréscimo em 16\% e inalterado em 45\%. O insucesso no aumento de PFMPN pode ser explicado pelo inadequado avanço dos maxilares ${ }^{5}$ e por movimento de intrusão ou reposicionamento inferior. Nos casos de pacientes portadores da Síndrome da Apnéia do Sono, este movimento deve ser próximo de $10 \mathrm{~mm}$ para aumentar o espaço aéreo posterior ${ }^{22}$.

A rotação cirúrgica anti-horária, não apenas de mandíbula, mas também de maxila, tem sido realizadas com sucesso para obter aumento máximo em pacientes com SAOS ${ }^{15}$. Segundo Liukkonen et al. ${ }^{13}$, é evidente que o recuo mandibular com rotação posterior pode gerar estreitamento do espaço aéreo posterior e ser fator contribuinte no desenvolvimento gradual de desordem respiratória. Isto pode ocorrer se houver fatores predisponentes, como um tipo craniofacial específico e/ou obesidade presente, ou se a adaptação neuromuscular for insuficiente para compensar a redução no tamanho do espaço.

De acordo com Gonçalves et al. ${ }^{8}$, que avaliaram as mudanças no espaço aéreo orofaríngeo após o avanço cirúrgico maxilomandibular com rotação anti-horária, o avanço dos maxilares com giro antihorário do plano oclusal melhora as dimensões do espaço aéreo orofaríngeo.

No presente estudo, o aumento de PFM-PN foi obtido com média de movimento mandibular de $8 \mathrm{~mm}$ (houve giro anti-horário em 33,3\% dos casos de avanço bimaxilar) e maxilar de $6 \mathrm{~mm}$. O valor de pm-PFS permaneceu constante, independente de o avanço ser mandibular ou bimaxilar. $\mathrm{Na}$ avaliação de Gonçalves et al. ${ }^{8}$, pm-PFS aumentou, enquanto o espaço aéreo orofaríngeo se manteve estável durante o período pós-cirúrgico.

A análise cefalométrica das medidas esqueléticas N-S-Ba e Ar-Go-Me, neste estudo, revelou que seus valores se elevaram significantemente $(73,3 \%$ e $60 \%$, respectivamente), apenas com o avanço mandibular. Para Moss ${ }^{16}$, quando a função muscular é alterada, normalmente associada à ação dos músculos masseter e pterigóideo medial, a morfologia do ângulo gonial se altera.

Conforme identificado no gráfico 1 , as alterações nas medidas cefalométricas deste estudo ocorreram mais em casos de avanço bimaxilar, podendo estar relacionadas ao giro anti-horário e à maior quantidade de tecido mole do espaço aéreo faríngeo envolvido neste tipo de avanço cirúrgico, que foi posicionado anteriormente. É válido ressaltar que o conhecimento do tamanho normal deste espaço deve ser de grande importância quando os- 
teotomias são planejadas, evitando-se possível desenvolvimento de desordem respiratória do sono.

\section{CONCLUSÃO}

Com base na metodologia empregada, foi possível observar que:

1) não ocorreu redução do espaço aéreo faríngeo após intervenção cirúrgica;
2) houve aumento significativo do espaço aéreo faríngeo, independente do tipo de avanço cirúrgico;

3) não se observou alteração esquelética (ArGo-Me e N-S-Ba) nos casos tratados com cirurgia de maxila e mandíbula.

\title{
Cephalometric evaluation of posterior air space in occlusion Class II patients treated with orthognatic surgery
}

\begin{abstract}
Aim: Maxillomandibular advancement is a surgical treatment for patients suffering from obstructive sleep apnea syndrome (OSAS) and those with identificable anatomic abnormalities in this complex that narrows and/or obstructs the airway. The purpose of this study is to evaluate pharyngeal airway space cephalometric variations in patients affected by Class II, treated with orthognatic surgery. Methods: The sample consisted of pre and postoperative (minimum 6 months) lateral radiographs of 30 patients, that had undergone mandibular $(n=15)$ and maxillomandibular ( $n=15)$ advancement. The cephalometric parameters used allowed to evaluate the posterior airway space at three levels: the hypopharynx (V-LPW), the oropharynx (PAS-MP, PAS-OP, PAS-U and PAS-Up) and the nasopharynx (PAS-NP, pm-UPW). The skeletal analyse was in cranial base (N-S-Ba) and in mandible (Ar-Go-Me). The mean difference between the pre and postoperative linear $(\mathrm{mm})$ and angular (degree) measurements was evaluated by paired $t$ test. Results and Conclusions: Statistically, there was no pharynx airway space reduction after surgery advancement. It was noted that only PAS-OP and pm-UPW remained constant and the values increased, for the most remaining part.
\end{abstract}

Key words: Cephalometry. Nasopharyngeal cavity. Class II occlusion. Orthognatic surgery.

\section{REFERÊNCIAS}

1. BATTAGEL, J. M.; L'ESTRANGE, P. R. The cephalometric morphology of patients with obstructive sleep apnoea (OSA). Eur. J. Orthod., Oxford, v. 18, p. 557-567, 1996.

2. BATTAGEL, J. M. et al. A cephalometric comparison of subjects with snoring and obstructive sleep apnoea. Eur. J. Orthod., Oxford, v. 22, p. 353-365, 2000.

3. BEAR, S. E.; PRIEST, J. H. Sleep apnea syndrome: correction with surgical advacement of the mandible. J. Oral Surg., Chicago, v. 38, no. 7, p. 543-549, 1980.

4. De BERRY-BOROWIECKI, B. et al. Cephalometric analysis for diagnosis and treatment of obstructive sleep apnea. Laryngoscope, Philadelphia, v. 98, p. 226-234, 1988

5. De PONTE, et al. Cephalometric study of posterior airway space in patients affected by Class II occlusion and treated with orthognathic surgery. J. Craniofacial Surg., Boston, v. 10, p. 252-259, May 1999.

6. GILON, Y. et al. Surgical management of maxillomandibular advancement in sleep apnea patients: specific technical considerations. Int. J. Adult Orthod. Orthognath. Surg., Chicago, v. 16, no. 4 , p. 305-314, 2001

7. GOODDAY, R. H. B. et al. Obstructive sleep apnea syndrome: diagnosis and management. J. Can. Dent. Assoc., Ottawa, v. 67, no. 11, p. 652-658, 2001.
8. GONÇALVES, J. R.; GONÇALVES, D. G.; WOLFORD, L. M. Postsurgical stability of oropharyngeal airway changes following counter-clockwise maxillo-mandibular advancement surgery. J. Oral Maxillofac. Surg., Philadelphia, v. 64, p. 755762, 2006.

9. HELLSING, E. Changes in the pharyngeal airway in relation to extension of the head. Eur. J. Orthod., Oxford, v. 11, p. 359, 1989.

10. HIERL, T. et al. Obstructive sleep apnoea syndrome: results and conclusions of a principal component analysis. J. Craniomaxillofac. Surg., Edinburgh, v. 25, p. 181-185, 1997.

11. HOCHBAN, W. et al. Surgical treatment of obstructive sleep apnea by maxillomandibular advancement. Sleep, Winchester, v. 17, p. $624-629,1994$

12. JAMIESON, A. et al. Obstructive sleep apnoea patients have craniomandibular abnormalities. Sleep, Winchester, v. 9 p. 469-477, 1986.

13. LIUKKONEN, M. et al. Effect of mandibular setback surgery on the posterior airway size. Int. J. Adult Orthod. Orthognath. Surg., Chicago, v. 17, no. 1, p. 441-416, 2002.

14. LOWE, A. A. et al. Cephalometric comparisons of craniofacial and upper airway structure by skeletal subtype and gender in patients with obstructive sleep apnea. Am. J. Orthod. Dentofacial Orthop., St. Louis, v. 110, p. 653-664, 1996. 
15. MEHRA, P. et al. Pharyngeal airway space changes after counterclockwise rotation of the maxillomandibular complex. Am. J. Orthod. Dentofacial Orthop., St. Louis, v. 120, p. 154-159, 2001.

16. MOSS, M. Functional analysis of human mandibular growth. J. Prosthet. Dent., St. Louis, v. 10, p. 1149-1159, 1960.

17. MUTO, T. et al. Relationship between the pharyngeal airway space and craniofacial morphology, taking into account head posture. Int. J. Oral Maxillofac. Surg., Copenhagen, v. 35 , p. 132-136, 2006

18. NAKAGAWA, F. et al. The effect of head posture on the pharyngeal airway space (PAS). Int. J. Adult Orthod. Orthognath. Surg., Chicago, v. 31, p. 579, 2002.

19. REMMERS, J. E. et al. Pathogenesis of upper airway occlusion during sleep. J. Appl. Physiol., Washington, D. C., v. 44, no. 8 , p. 931-938, 1978

20. REICHE-FISCHEL, O.; WOLFORD, L. M. Posterior airway space changes after double jaw surgery with counter-clockwise rotation. AAOMS 78th Annual Meeting and Scientific Sessions. J. Oral Maxillofac. Surg., Philadelphia, v. 54, p. 96, 1996.

21. RILEY, R. W. et al. Obstructive sleep apnea syndrome following surgery for mandibular prognathism. J. Oral Maxillofac. Surg. Philadelphia, v. 45, p. 450-452, 1987.

22. RILEY, R. et al. Obstructive sleep apnea syndrome: a surgical protocol for dynamic upper airway reconstruction. J. Oral Maxillofac. Surg., Philadelphia, v. 51, p. 742-747, 1993.
23. SCHENDEL, S. A. et al. Velopharyngeal anatomy and maxillary advancement. J. Oral Maxillofac. Surg., Philadelphia, v. 7 p. 116-124, 1979

24. SCHWAB, R. J.; GOLDBERT, A. N. Upper airway assessment: radiographic and other imagining techniques. Otolaryngol. Clin. North Am., Philadelphia, v. 31, no. 6, p. 931-968, 1998.

25. SHEPARD JR., J. W. Hipertension, cardiac arrhythmias, myocardial infarction, and stroke in relation to obstructive sleep apnea. Clin. Chest Med., Philadelphia, v. 13, no. 3, p. 437-458, 1992.

26. SOLON, B. et al. Airway dimensions and head posture in obstructive sleep apnoea. Eur. J. Orthop., Oxford, v. 18 p. $571-579,1996$

27. TANGUGSORN, V. et al. Obstructive sleep apnea: a cephalometric study. Part I. Cervico-craniofacial skeletal morphology. Eur. J. Orthop., Oxford, v. 17, p. 45-56, 1995

28. TANGUGSORN, V. et al. Obstructive sleep apnea: a cephalometric study. Part II. Uvulo-glossopharyngeal morphology. Eur. J. Orthop., Oxford, v. 17, p. 57-67, 1995.

29. TUMBULL, N. R.; BATTAGEL, J. M. The effects of orthognathic surgery on pharyngeal airway dimensions and quality of sleep. J. Orthod., London, v. 27, p. 235-247, 2000.

30. TSUCHIYA, M. et al. Obstructive sleep apnea subtypes by cluster analysis. Am. J. Orthod. Dentofacial Orthop., St. Louis, v. 101, p. $533-542,1992$
Endereço para correspondência

Valfrido Antonio Pereira Filho

Rua Humaitá, $16802^{\circ}$ andar Centro

CEP: 14.801-903 - Araraquara / SP

E-mail: dinho@foar.unesp.br 